\title{
ELECTRORETINOGRAPHIC FINDINGS IN DAY-BLIND DOGS
}

\author{
Magdalena DrĄŻEK-KUBIAK ${ }^{1}$, Marcin LEW $^{1 *}$ and Sylwia LEW ${ }^{2}$ \\ ${ }^{1}$ Department of Surgery and Radiology, Faculty of Veterinary Medicine and \\ ${ }^{2}$ Department of Microbiology, Faculty of Biology and Biotechnology, University of \\ Warmia and Mazury, ul. M. Oczapowskiego 14, 10-719 Olsztyn, Poland
}

(Received 2 October 2017; accepted 16 April 2018)

\begin{abstract}
Cone degeneration (cd; day blindness) is one of the inherited retinal diseases of dogs. Its diagnosis is based on vision testing, fundoscopy, electroretinography (ERG) and, for some breeds, on genetic tests. Typical signs of the disease are day blindness and cone dysfunction during ERG while fundoscopy does not show any abnormalities. The aim of this study was to compare behavioural findings, fundoscopic lesions and electroretinographic alterations in 12 cd-affected dogs (Alaskan Malamute, Labrador Retriever, German Shepherd, Dachshund, Yorkshire Terrier, Shih Tzu, Siberian Husky and crossbreeds) examined at our clinic. None of the examined dogs had any fundoscopic lesions, and all of them had normal scotopic vision with strongly impaired or absent photopic vision. Light-adapted transient, cone-mediated and steady-state, $31-\mathrm{Hz}$ cone flicker ERGs were much below the 5th percentile limits of normality or even unrecordable, while the rod-driven ERGs were within normal values. Vision test and ERG results corresponded to each other and, combined with the results of fundoscopy, were typical of cd. To date, our research is one of the few studies in the world presenting ERG alterations compared with vision test findings and fundoscopic results in the course of $\mathrm{cd}$.
\end{abstract}

Key words: Electroretinogram, day blindness, inherited eye diseases, cone degeneration, dog

Cone degeneration (cd), also known as achromatopsia (day blindness) or hemeralopia, represents a group of inherited retinal disorders (Rubin et al., 1967; Narfström and Petersen-Jones, 2013). The essence of the disease is the progressive degeneration of normally developed cones, while rod function remains unaltered (Koch and Rubin, 1971; Rubin, 1971a,b; Aguirre and Rubin, 1974, 1975; Gropp et al., 1996; Michaelides et al., 2006; Narfström and Petersen-Jones, 2013).

Electron microscopic studies revealed disorganisation of lamellar discs in the cone outer segments. In the inner segments, accumulations of filamentcontaining bundles, displaced cone nuclei and a reduction in their size by $2 / 3$ are seen (Aguirre and Rubin, 1974). It has been shown that the number of cones be-

"Corresponding author; E-mail: lew@uwm.edu.pl; Phone/Fax: 0048 (89) 523-4178 
comes reduced at the age of 4 years when the retina in cd-affected dogs is made only of rods (Aguirre and Rubin, 1974; Long and Aguirre, 1991). Gropp et al. (1996) demonstrated that in cd the cones die due to a displacement of the nucleus into the interphotoreceptor space, which may be related to the lack of $\beta 3$ transducine expression.

The disease can be diagnosed from 7-12 weeks of age (Rubin, 1971a,b; Aguirre and Rubin, 1974; Narfström and Petersen-Jones, 2013). Day blindness is a typical sign (Rubin, 1971a,b; Aguirre and Rubin, 1974; Hurn et al., 2003; Narfström and Petersen-Jones, 2013; Dixon, 2016). Under photopic conditions, the dog becomes insecure, moves more carefully and appears to be blind (Rubin, 1971a,b; Hurn et al., 2003; Narfström and Petersen-Jones, 2013; Dixon, 2016). Additionally, under light conditions, dogs with cd can be photophobic, and some of them can show miosis (Rubin, 1971a,b; Hurn et al., 2003, Dixon, 2016). Under mesopic and scotopic conditions, the animal acts normally and avoids obstacles, which it hits during daylight hours (Rubin, 1971a,b; Hurn et al., 2003). Throughout life, in animals with cd no fundoscopic lesions are observed and pupillary light reflexes (PLR) are normal under all light conditions (Rubin, 1971a,b; Narfström and Petersen-Jones, 2013). The results of electroretinography (ERG) in the course of cd demonstrate normal values for rod-driven responses, while cone-driven responses are markedly reduced or even absent (Aguirre and Rubin, 1975; Andréasson and Tornqvist, 1991; Hurn et al., 2003; Shamir et al., 2010; Genead et al., 2011; Narfström and Petersen-Jones, 2013; Dixon, 2016).

Due to the normal fundus appearance, the diagnosis of cd is based on the observation of the animal's behaviour under different light intensity conditions and on ERG (Narfström and Petersen-Jones, 2013). For selected canine breeds, the diagnosis of cd is aided by genetic tests. As far as the aetiology of cd is concerned, CNGA3 (Cyclic Nucleotide Gated Channel Alpha 3) gene mutation in German Shepherd (R424W mutation) and Labrador Retriever (V644del mutation) (Tanaka et al., 2015), CNGB3 (Cyclic Nucleotide Gated Channel Beta 3) gene mutations in German Shorthaired Pointers (D262N mutation) (Sidjanin et al., 2002) and CNGB3 gene deletion in Alaskan Malamute (Sidjanin et al., 2002), Miniature Australian Shepherd, Alaskan Sled Dogs and Siberian Husky (Yeh et al., 2013) have been discussed. All these genes mutations are specifically expressed in the cone photoreceptors leading to achromatopsia.

Single cases of cd have been diagnosed in Miniature Poodle (Rubin, 1989), Australian Cattle Dog, Chihuahua, Rhodesian Ridgeback (Hurn et al., 2003), Wirehaired Dachshund (Ropstad et al., 2005) and German Wirehaired Pointer (McElroy, 2006).

An increasing number of cases presenting the prevalence of $\mathrm{cd}$ in different canine breeds suggests that the disease has a wider range than has been described. From the clinical point of view, the development of reliable and accurate meth- 
ods enabling the detection of cd in dogs regardless of the breed would be very useful, especially in dog breeds in which genetic tests have not been developed.

The aim of this study was to compare behavioural findings, fundoscopic lesions and electroretinographic alterations in $12 \mathrm{~cd}$-affected dogs examined at our clinic.

\section{Materials and methods}

This study was conducted at our clinic during a 4-year period (20112015), on 12 pedigree and crossbreed dogs ( 24 eyes with cd), both sexes in the age ranged from 14 weeks to 9 years. Three of them were crossbreeds ( 5 months, 1 year, 9 years of age), while the others represented seven breeds: one Labrador Retriever (5 years of age), two Alaskan Malamutes (6 months, 7 years of age), one Yorkshire Terrier (3 years of age), one German Shepherd (9 months old), one Dachshund (14 weeks old), two Shih Tzu (4 and 8 years of age) and one Siberian Husky (7 years of age). The owners told us that the dogs had vision problems in the daylight hours, e.g. they collided with obstacles and showed photophobia. These dogs constituted the experimental group (G1).

The ophthalmic examination included a maze test, menace response and dazzle reflex, under photopic and scotopic conditions as well as direct and consensual PLR and fundoscopy.

The examination procedure was completed including digital slit-lamp biomicroscopy (Hawk Eye, Dioptrix, France), indirect and direct ophthalmoscopy (Omega 500, Heine Instruments, Germany; PanOptic Ophthalmoscope, Welch Allyn, USA), gonioscopy (Koeppe Goniolens, Ocular Instruments, inc., USA) and tonometry (Icare Tonovet, USA). Electroretinography was performed using two channels EasyVEP (Acrivet, Germany). Active, Kooyman-Damhof electrode (Acrivet, Germany) with an LED Flash $4 \mathrm{~W}$ generator and $0.3 \mathrm{~mm}$ stainless steel, subdermal needle, reference and ground electrodes (Acrivet, Germany) were used in the examination. The evaluation of rod-driven, mixed rod-cone and cone-driven responses was carried out according to the guidelines for clinical ERG in the dog (rod-driven responses during dark adaptation at 0, 4, 8, 12, 16 and $20 \mathrm{~min}$, respectively - single flash of light for each measurement, the stimulus intensity $0.01 \mathrm{~cd} \mathrm{~s} \mathrm{~m}^{-2}$; dark adapted, mixed rod-cone response - two stimuli were averaged with a 15 -second interval, stimulus intensity $3 \mathrm{~cd} \mathrm{~s} \mathrm{~m}^{-2}$; light adaptation time - $10 \mathrm{~min}$ with white background light of $30 \mathrm{~cd} \mathrm{~m}^{-2}$, stimulus intensity $3 \mathrm{~cd} \mathrm{~s} \mathrm{~m}^{-2}$ - three responses were averaged; cone flicker $31 \mathrm{~Hz}$, the same light conditions) (Ekesten et al., 2013).

Electroretinography was performed under general anaesthesia with the standards of our laboratory (Drążek et al., 2016). Atropine sulphate (Atropinum sulfuricum WZF, Polfa, Poland) at a dose of $0.04 \mathrm{mg} / \mathrm{kg}$ body weight (BW) and xylazine (Vetaxyl, VetAgro, Poland) at a dose of $2 \mathrm{mg} / \mathrm{kg} \mathrm{BW}$, administered i.m. 
were used for premedication. General anaesthesia was provided with xylazine (Vetaxyl, VetAgro, Poland) at a dose of $2 \mathrm{mg} / \mathrm{kg}$ BW and ketamine (Vetketam, VetAgro, Poland) at a dose of $5 \mathrm{mg} / \mathrm{kg} \mathrm{BW}$, administered i.v. Mydriasis was achieved with $0.5 \%$ tropicamide (Mydriacyl, Alcon, Poland). GonioVisc (HUB Pharmaceuticals, USA) was administrated between the corneal surface and the active electrode.

In the control group (G0), the implicit time and amplitude baseline values were based on the averages of 50 results randomly chosen from the database of the Clinic, obtained during screening examinations of healthy dogs with ages corresponding to those of the dogs in the experimental group.

\section{Results}

A total of 12 dogs demonstrated a loss of vision under daylight conditions and three of them had some signs of miosis (Table 1). During a maze test performed under photopic conditions, 9 dogs collided with obstacles and seemed to be completely blind. Seven of them were photophobic. The other 3 animals were sometimes able to pass between some objects. Under scotopic conditions, all dogs were more active and avoided obstacles. Menace response and dazzle reflex in all light conditions as well as direct and consensual PLR were normal in both eyes. All of the examined dogs had a normal appearance of anterior segment, iridocorneal angle and fundus (Figs 1 and 2) and their intraocular pressure values were in the reference range $(15-25 \mathrm{~mm} \mathrm{Hg})$.

The results of the 12 ERG examinations are presented in Fig. 3 in the order corresponding to Table 1. The cone-mediated ERGs in all investigated dogs were much below 5 th percentiles while the rod-driven responses were within normal values. Cone-mediated ERGs for cases 5, 6 and 10 had a recognisable morphology but their amplitudes were also slightly below 5 th percentiles.

\section{Discussion}

Electroretinography allows earlier recognition of retinal diseases than a routine ophthalmic examination (Ekesten, 2013; Narfström and Petersen-Jones, 2013; Drążek et al., 2016), although the time of a correct diagnosis may vary significantly for the individual disorders (Drążek et al., 2014, 2016). Moreover, for cd and e.g. congenital stationary night blindness, which do not present any ophthalmoscopic changes throughout the entire life of the affected dogs, ERG is the only objective method to diagnose the condition (Nilsson et al., 1992; Narfström et al., 1994). The changes in the dog's behaviour under variable light intensity are the only clinical signs that may indicate cd. 
Table 1

Results of the ophthalmic examination

\begin{tabular}{|c|c|c|c|c|c|c|c|c|c|c|c|}
\hline & & \multicolumn{5}{|c|}{ Photopic } & \multicolumn{5}{|c|}{ Scotopic } \\
\hline & & 1 & 2 & 3 & 4 & 5 & 1 & 2 & 3 & 4 & 5 \\
\hline Labrador Retriever & OD & \multirow{2}{*}{-} & - & - & ++ & ++ & \multirow{2}{*}{++} & ++ & ++ & ++ & ++ \\
\hline 5 y., male $(\mathrm{P}, \mathrm{M})$ & OS & & - & - & ++ & ++ & & ++ & ++ & ++ & ++ \\
\hline Alaskan Malamute & OD & \multirow[b]{2}{*}{-} & - & - & ++ & ++ & \multirow{2}{*}{++} & ++ & ++ & ++ & ++ \\
\hline 7 y., male $(\mathrm{P})$ & OS & & - & - & ++ & ++ & & ++ & ++ & ++ & ++ \\
\hline Yorkshire Terrier & OD & \multirow{2}{*}{-} & - & - & ++ & ++ & \multirow{2}{*}{++} & ++ & ++ & ++ & ++ \\
\hline 3 y., male & OS & & - & - & ++ & ++ & & ++ & ++ & ++ & ++ \\
\hline German Shepherd & OD & \multirow[b]{2}{*}{-} & - & - & ++ & ++ & \multirow{2}{*}{++} & ++ & ++ & ++ & ++ \\
\hline 9 m., female $(\mathrm{P})$ & OS & & - & - & ++ & ++ & & ++ & ++ & ++ & ++ \\
\hline Alaskan Malamute & OD & \multirow{2}{*}{+} & - & - & ++ & ++ & \multirow{2}{*}{++} & ++ & ++ & ++ & ++ \\
\hline $6 \mathrm{~m} .$, male & OS & & - & - & ++ & ++ & & ++ & ++ & ++ & ++ \\
\hline Dachshund & OD & \multirow{2}{*}{+} & - & - & ++ & ++ & \multirow{2}{*}{++} & ++ & ++ & ++ & ++ \\
\hline 14 w., male & OS & & - & - & ++ & ++ & & ++ & ++ & ++ & ++ \\
\hline Shih Tzu & $\mathrm{OD}$ & \multirow[b]{2}{*}{-} & - & - & ++ & ++ & \multirow{2}{*}{++} & ++ & ++ & ++ & ++ \\
\hline 4 y., female & OS & & - & - & ++ & ++ & & ++ & ++ & ++ & ++ \\
\hline Siberian Husky & OD & \multirow{2}{*}{-} & - & - & ++ & ++ & \multirow{2}{*}{++} & ++ & ++ & ++ & ++ \\
\hline 7 y., female $(P, M)$ & OS & & - & - & ++ & ++ & & ++ & ++ & ++ & ++ \\
\hline Shih Tzu & OD & \multirow{2}{*}{-} & - & - & ++ & ++ & \multirow{2}{*}{++} & ++ & ++ & ++ & ++ \\
\hline 8 y., female $(P)$ & OS & & - & - & ++ & ++ & & ++ & ++ & ++ & ++ \\
\hline Crossbreed 1 & OD & \multirow{2}{*}{+} & - & - & ++ & ++ & \multirow{2}{*}{++} & ++ & ++ & ++ & ++ \\
\hline $5 \mathrm{~m} .$, male & OS & & - & - & ++ & ++ & & ++ & ++ & ++ & ++ \\
\hline Crossbreed 2 & OD & \multirow{2}{*}{-} & - & - & ++ & ++ & \multirow{2}{*}{++} & ++ & ++ & ++ & ++ \\
\hline 1 y., male $(\mathrm{P})$ & OS & & - & - & ++ & ++ & & ++ & ++ & ++ & ++ \\
\hline Crossbreed 3 & OD & \multirow{2}{*}{-} & - & - & ++ & ++ & \multirow{2}{*}{++} & ++ & ++ & ++ & ++ \\
\hline 9 y., female $(P, M)$ & OS & & - & - & ++ & ++ & & ++ & ++ & ++ & ++ \\
\hline
\end{tabular}

y: year(s) old; m: month(s) old, w: week(s) old; 1: maze test, 2: menace response, 3: dazzle reflex, 4: direct pupillary light reflex (PLR), 5: consensual PLR. Results are described as normal $(++)$, reduced $(+)$, absent $(-)$; OD: right eye, OS: left eye, P: photophobia, M: miosis

Day blindness and the lack of fundoscopic lesions raise the suspicion of cd (Narfström and Petersen-Jones, 2013). However, an early-stage cone rod dystro- 
phy (crd) may present the same behavioural signs and a lack of fundoscopic changes, in some cases even up to 3 years of age (Ropstad et al., 2007a). The diagnosis of cd should thus be confirmed with an ERG that determines the type and degree of severity of retinal lesions.

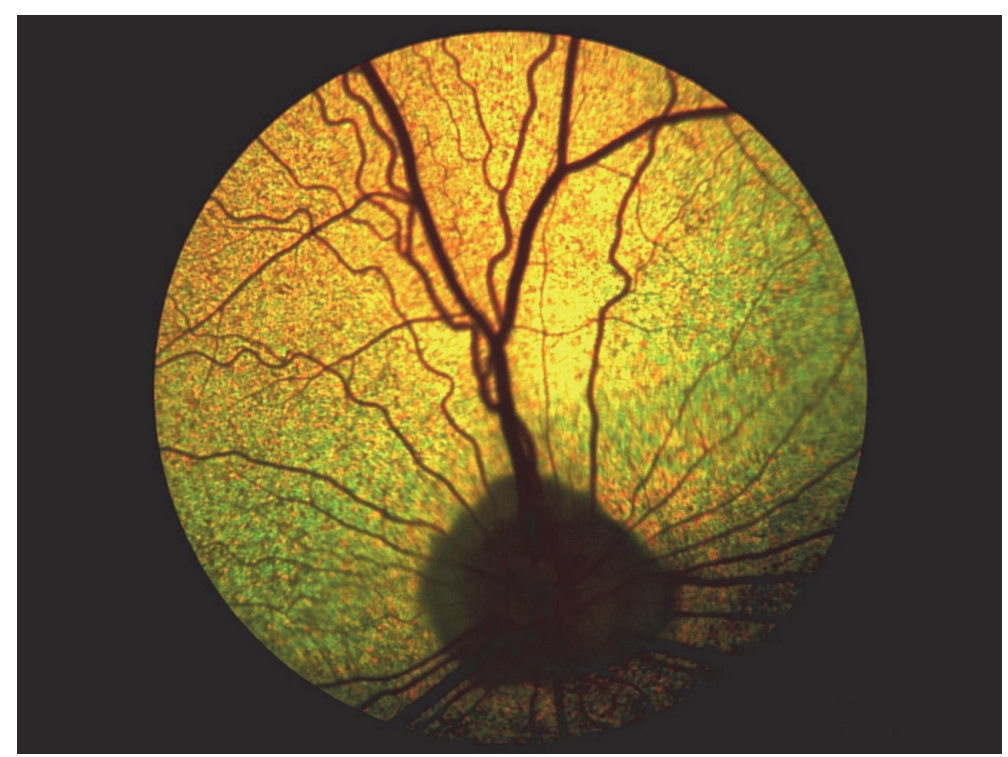

Fig. 1. Fundus appearance of 5-year-old male Labrador Retriever



Fig. 2. Fundus appearance of 1-year-old male Crossbreed 2 


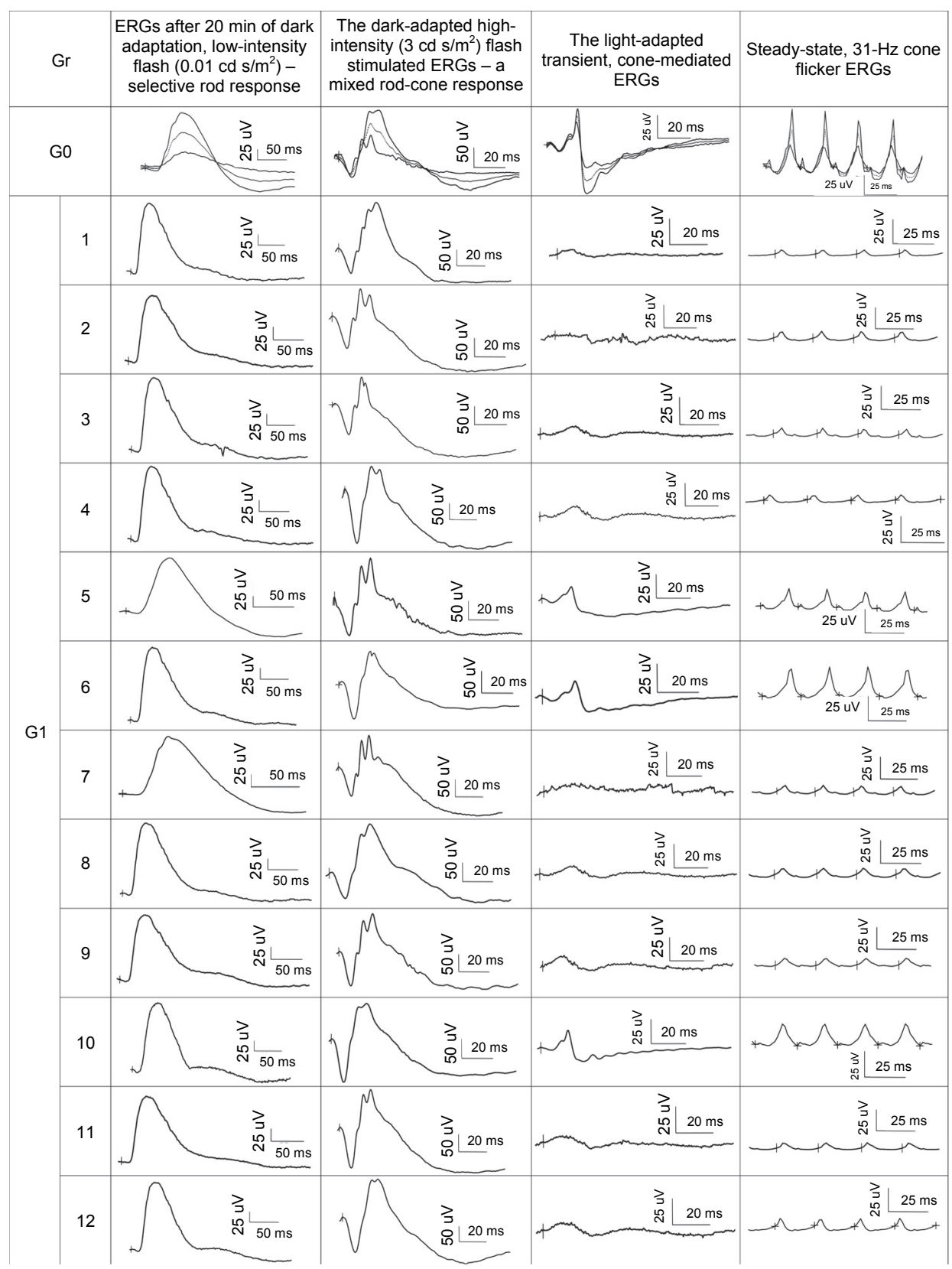

Fig. 3. ERGs from control group (G0) and dogs included in the study (G1). Group G0 consists of median (dotted line) and limits of normality using the 5th and 95th percentiles (solid line) according to the guidelines recommended by Ekesten et al. (2013). Group G1 consists of individual ERG traces of 12 dogs (1 to 12) in the order corresponding to Table 1. (1) Labrador Retriever, 5-year-old male; (2) Alaskan Malamute, 7-year-old male; (3) Yorkshire Terrier, 3-year-old male; (4) German Shepherd, 9-month-old female; (5) Alaskan Malamute, 6-month-old male; (6) Dachshund, 14-week-old male; (7) Shih Tzu, 4-year-old female; (8) Siberian Husky, 7-year-old female;

(9) Shih Tzu, 8-year-old female; (10) Crossbreed 1, 5-month-old male; (11) Crossbreed 2, 1-year-old male; (12) Crossbreed 3, 9-year-old female. The calibration bars are denoted separately for each type of ERG response. Markers "|" indicate the onset of a light stimulus. Rod-driven responses during dark adaptation at 0, 4, 8, 12,16

and $20 \mathrm{~min}$, respectively - single flash of light for each measurement, stimulus intensity $0.01 \mathrm{~cd} \mathrm{~s} \mathrm{~m}^{-2}$;

dark adapted, mixed rod-cone response - two stimuli were averaged with a 15 -second interval, stimulus intensity $3 \mathrm{~cd} \mathrm{~s} \mathrm{~m}^{-2}$; light adaptation time $-10 \mathrm{~min}$ with white background light of $30 \mathrm{~cd} \mathrm{~m}^{-2}$, stimulus intensity $3 \mathrm{~cd} \mathrm{~s} \mathrm{~m}^{-2}-$ three responses were averaged; cone flicker $31 \mathrm{~Hz}$, the same light conditions 
The 12 dogs included in this study had similar vision testing results to cdaffected dogs (Aguirre and Rubin, 1974; Hurn et al., 2003; Narfström and Petersen-Jones, 2013; Dixon, 2016). As in other reports, among these dogs there were Labrador Retriever (Dixon, 2016) and Alaskan Malamute (Koch and Rubin, 1971; Rubin, 1971a,b) as well as German Shepherd, Dachshund, Yorkshire Terrier, Shih Tzu, Siberian Husky and crossbreed dogs. Under daylight conditions, 9 dogs moved cautiously and collided with obstacles, whereas in dim light they were more active and avoided barriers. Seven out of nine dogs demonstrated photophobia, of which 3 had miosis, which may occur in cd (Rubin, 1971a,b; Hurn et al., 2003; Dixon, 2016).

In the examined group, 3 dogs (14 weeks, 5 months and 6 months of age) were sometimes able to avoid some objects in daylight. Vision testing can be a quite imprecise examination due to a multitude of factors unrelated to retinal function, which potentially affect the results (e.g. the other senses or simply an accidental situation) (Narfström and Petersen-Jones, 2013). Apart from cd, such signs may be demonstrated in the initial stage of crd, when a progressive degeneration of cones and rods occurs, although the cone system is affected earlier and more severely (Ropstad, 2007b; Narfström and Petersen-Jones, 2013). In the case of crd, affected puppies show miosis under light conditions (Ropstad et al., 2007a) and the fundoscopic lesions appear at the age of 3-6 months (Narfström and Petersen-Jones, 2013).

In cd, ERG alterations are only limited to the cone responses (Aguirre and Rubin, 1975; Andréasson and Tornqvist, 1991; Hurn et al., 2003; Shamir et al., 2010; Genead et al., 2011; Narfström and Petersen-Jones, 2013; Dixon, 2016). It has been shown that the function and number of cones decrease with age (Aguirre and Rubin, 1974; Long and Aguirre, 1991). Initially, at 3-6 weeks of age ERGs may be normal and then they may start to indicate a reduction or absence of the cone function with normal rod responses (Aguirre and Rubin, 1974; Aguirre and Rubin, 1975; Long and Aguirre, 1991; Narfström and Petersen-Jones, 2013). Electron microscopic examinations confirm a progressive reduction of cone number with age (Aguirre and Rubin, 1974; Komáromy et al., 2010).

The ERG results of the 12 dogs correlated with the findings of vision tests. The vision test results clearly indicated that all of the examined dogs had normal scotopic vision and a strongly impaired or absent day vision, and thus were typical of cd (Aguirre and Rubin, 1975; Andréasson and Tornqvist, 1991; Hurn et al., 2003; Shamir et al., 2010; Genead et al., 2011; Narfström and Petersen-Jones, 2013; Dixon, 2016). Like in the studies of Hurn et al. (2003) and Dixon (2016), the pure rod responses were normal in all examined dogs and they were in the range between 5 and 95 percentiles of the control group, whereas the lightadapted transient, cone-mediated and steady-state, $31-\mathrm{Hz}$ cone flicker ERGs were significantly reduced. In our research the cone-driven ERGs no. 5, 6 and 10 (Fig. 3), obtained accordingly from a 6-month-old Alaskan Malamute, a 14- 
week-old Dachshund and a 5-month-old crossbreed, which were sometimes able to avoid some objects in daylight, were slightly below the 5th percentile limits of normality (Fig. 3). The cone-driven ERG results of the remaining 9 dogs were unrecordable. Especially $31-\mathrm{Hz}$ cone flicker ERGs exhibited peaks in the form of artefacts (Fig. 3).

In crd, cone-specific ERGs are reduced and rod responses are normal at the age of 5-6 weeks (Ropstad et al., 2007b) and by 12-15 months of age both are non-recordable (Narfström and Petersen-Jones, 2013). Due to the similarity of behavioural changes, ophthalmic signs and electroretinographic findings in cd and in the initial stage of crd, the final diagnosis for the three above-mentioned dogs (ages: 14 weeks, 5 and 6 months) should be established after the second ERG examination at the age of 15 months. Furthermore, in the 14-week-old Dachshund the probability of crd is higher than that of cd (Ropstad et al., 2007a,b; Narfström and Petersen-Jones, 2013), while in the 6-month-old Alaskan Malamute cd is more probable (Rubin et al., 1967; Rubin, 1971a,b; Aguirre and Rubin, 1974; Narfström and Petersen-Jones, 2013).

Dogs with any opacity of the optic system were excluded from this study. In the studies by Aguirre and Rubin (1974), no other ocular abnormalities were recorded in dogs with cd, whereas in Dixon's studies (2016) cd was accompanied by immature diffuse posterior cataract in one dog. In our opinion, vision testing is an important part of the diagnosis of $\mathrm{cd}$, and thus the material selection should have excluded dogs with any coinciding visual opacity that may interfere with the results.

Electroretinography performed before week 12 of life may not have any diagnostic value due to the ongoing retinal maturation that occurs between 8 and 12 weeks of life (Gum et al., 1984; Martin, 2010; Cook, 2013). Accordingly, only dogs over 3 months of age were included in the current study.

None of the examined dogs had any fundoscopic lesions, while visual alterations under photopic conditions corresponded to the ERG changes. The prevalence of $\mathrm{cd}$ in individual dog breeds and the nature of this disease need further study on a larger volume of clinical material.

\section{References}

Aguirre, G. D. and Rubin, L. F. (1974): Pathology of hemeralopia in the Alaskan malamute dog. Invest. Ophthalmol. Vis. Sci. 13, 231-235.

Aguirre, G. D. and Rubin, L. F. (1975): The electroretinogram in dogs with inherited cone degeneration. Invest. Ophthalmol. Vis. Sci. 14, 840-847.

Aguirre, G. D., Rubin, L. F. and Bistner, S. I. (1972): Development of the canine eye. Am. J. Vet. Res. 33, 2399-2414.

Andréasson, S. and Tornqvist, K. (1991): Electroretinograms in patients with achromatopsia. Acta Ophthalmol. Copenh. 69, 711-716. 
Cook, C. S. (2013): Ocular embryology and congenital malformations. In: Gelatt, K. N., Gilger, B. C. and Kern, T. J. (eds) Veterinary Ophthalmology. Wiley-Blackwell, Ames, Iowa. pp. 3-38.

Dixon, C. J. (2016): Achromatopsia in three sibling Labrador Retrievers in the UK. Vet. Ophthalmol. 19, 68-72.

Drążek, M., Lew, M., Lew, S. and Pomianowski, A. (2014): Electroretinography in dogs: a review. Vet. Med. 59, 515-526.

Drążek, M., Lew, M., Lew, S., Snarska, A. and Sobiech, P. (2016): Electroretinographic examination for evaluation of retinal activity in dogs with retinal dysplasia. Vet. Med. 61, 204-212.

Ekesten, B. (2013): Electrodiagnostic evaluation of vision. In: Gelatt, K. N., Gilger, B. C. and Kern, T. J. (eds) Veterinary Ophthalmology. Wiley-Blackwell, Ames, Iowa. pp. 684-702.

Ekesten, B., Komaromy, A. M., Ofri, R., Petersen-Jones, S. M. and Narfström, K. (2013): Guidelines for clinical electroretinography in the dog: 2012 update. Doc. Ophthalmol. 127, 79-87.

Genead, M. A., Fishman, G. A., Rha, J., Dubis, A. M., Bonci, D. M. O., Dubra, A. E., Stone, E. M., Neitz, M. and Carroll, J. (2011): Photoreceptor structure and function in patients with congenital achromatopsia. Invest. Ophthalmol. Vis. Sci. 52, 7298-7308.

Gropp, K. E., Szél, A., Huang, J. C., Acland, G. M., Farber, D. B. and Aguirre, G. D. (1996): Selective absence of cone outer segment beta 3-transducin immunoreactivity in hereditary cone degeneration (cd). Exp. Eye Res. 63, 285-296.

Gum, G. G., Gelatt, K. N. and Samuelson, D. A. (1984): Maturation of the retina of the canine neonate as determined by electroretinography and histology. Am. J. Vet. Res. 45, 1166-1171.

Hurn, S. D., Hardman, C. and Stanley, R. G. (2003): Day-blindness in three dogs: clinical and electroretinographic findings. Vet. Ophthalmol. 6, 127-130.

Koch, S. A. and Rubin, L. F. (1971): Distribution of cones in the hemeralopic dog. J. Am. Vet. Med. Assoc. 159, 1257-1259.

Long, K. O. and Aguirre, G. D. (1991): The cone matrix sheath in the normal and diseased retina: cytochemical and biochemical studies of peanut agglutinin-binding proteins in cone and rod-cone degeneration. Exp. Eye Res. 52, 699-713.

Martin, L. C. (2010): Vitreous and ocular fundus. Diseases of the retina and choroid. In: Martin, L. C. (ed.) Ophthalmic Disease in Veterinary Medicine. Softcover ed., with revisions. Manson Publishing Ltd., London. pp. 413-448.

McElroy, R. P. (2006): Day blindness in a German wirehaired pointer. Vet. Rec. 158, 175.

Michaelides, M., Hardcastle, A. J., Hunt, D. M. and Moore, A. T. (2006): Progressive cone and cone-rod dystrophies: phenotypes and underlying molecular genetic basis. Surv. Ophthalmol. 51, 232-258.

Narfström, K. and Petersen-Jones, S. M. (2013): Diseases of the canine ocular fundus. In: Gelatt, K. N., Gilger, B. C. and Kern, T. J. (eds) Veterinary Ophthalmology. Wiley-Blackwell, Ames, Iowa. pp. 1303-1392.

Narfström, K., Wrigstad, A., Ekesten, B. and Nilsson, S. E. (1994): Hereditary retinal dystrophy in the Briard dog: Clinical and hereditary characteristics. Vet. Comp. Ophthalmol. 4, 85-92.

Nilsson, S. E., Wrigstad, A. and Narfström, K. (1992): Changes in the DC electroretinogram in Briard dogs with hereditary congenital night blindness and partial day blindness. Exp. Eye Res. 54, 291-296.

Ropstad, E. O., Bjerkas, E. and Narfström, K. (2005): Clinical and fundoscopic signs of early onset day blindness (hemeralopia) in Wirehaired Dachshunds. Abstract No. 9. Abstracts: European College of Veterinary Ophthalmologists and European Society of Veterinary Ophthalmology, Oporto, Portugal. Vet. Ophthalmol. 8, 427-436.

Ropstad, E. O., Bjerkas, E. and Narfström, K. (2007a): Clinical findings in early onset cone-rod dystrophy in the Standard Wire-haired Dachshund. Vet. Ophthalmol. 10, 69-75.

Ropstad, E. O., Bjerkas, E. and Narfström, K. (2007b): Electroretinographic findings in the Standard Wire-haired Dachshund with inherited early onset cone-rod dystrophy. Doc. Ophthalmol. 114, 27-36. 
Rubin, L. F. (1971a): Clinical features of hemeralopia in the adult Alaskan Malamute. J. Am. Vet. Med. Assoc. 158, 1696-1698.

Rubin, L. F. (1971b): Hemeralopia in Alaskan Malamute pups. J. Am. Vet. Med. Assoc. 158, $1699-1701$.

Rubin, L. F. (1989): Inherited Eye Diseases in Purebred Dogs. Williams \& Wilkins, Philadelphia, $130 \mathrm{pp}$.

Rubin, L. F., Bourns, T. K. and Lord, L. H. (1967): Hemeralopia in dogs: heredity of hemeralopia in Alaskan Malamutes. Am. J. Vet. Res. 28, 355-357.

Shamir, M. H., Ofri, R., Bor, A., Brenner, O., Reicher, S., Obolensky, A., Averbukh, E., Banin, E. and Gootwine, E. (2010): A novel day blindness in sheep: epidemiological, behavioural, electrophysiological and histopathological studies. Vet. J. 185, 130-137.

Sidjanin, D. J., Lowe, J. K., McElwee, J. L., Milne, B. S., Phippen, T. M., Sargan, D. R., Aguirre, G. D., Acland, G. M. and Ostrander, E. A. (2002): Canine CNGB3 mutations establish cone degeneration as orthologous to the human achromatopsia locus ACHM3. Hum. Mol. Genet. 11, 1823-1833.

Tanaka, N., Dutrow, E. V., Miyadera, K., Delemotte, L., MacDermaid, C. M., Reinstein, S. L., Crumley, W. R., Dixon, C. J., Casal, M. L., Klein, M. L., Aguirre, G. D., Tanaka, J. C. and Guziewicz, K. E. (2015): Canine CNGA3 gene mutations provide novel insights into human achromatopsia-associated channelopathies and treatment. PLoS One 10, e0138943.

Yeh, C. Y., Goldstein, O., Kukekova, A. V., Holley, D., Knollinger, A. M., Huson, H. J., PearceKelling, S. E., Acland, G. M. and Komaromy, A. M. (2013): Genomic deletion of CNGB3 is identical by descent in multiple canine breeds and causes achromatopsia. BMC Genet. $14,27$. 